\title{
Modeling the origin of functional heterogeneity among auditory nerve fibers
}

\author{
Nikolai M Chapochnikov*1,2,3,5, Thomas Frank1,2,5, Nicola Strenzke1, \\ Andreas Neef ${ }^{1,2,3}$, Darina Khimich ${ }^{1,2}$, Alexander Egner ${ }^{4}$, Fred Wolf2,3,5 and \\ Tobias Moser $1,2,5$
}

\begin{abstract}
Address: ${ }^{1}$ InnerEarLab, Department of Otolaryngology and Center for Molecular Physiology of the Brain, University of Göttingen, Germany, ${ }^{2}$ Bernstein Center for Computational Neuroscience, University of Göttingen, 37073, Germany, ${ }^{3}$ Department of Nonlinear Dynamics, Max-PlanckInstitute for Dynamics \& Self-Organization, Göttingen, Germany, ${ }^{4}$ Department of NanoBioPhotonics, MPI for Biophysical Chemistry, Göttingen, Germany and ${ }^{5}$ Göttingen Graduate School for Neurosciences and Molecular Biosciences (GGNB), Göttingen, Germany

Email: Nikolai M Chapochnikov* - nikolai@nld.ds.mpg.de

* Corresponding author
\end{abstract}

from Eighteenth Annual Computational Neuroscience Meeting: CNS*2009

Berlin, Germany. 18-23 July 2009

Published: 13 July 2009

BMC Neuroscience 2009, I0(Supp| I):P220 doi:I0.I|86/I47|-2202-I0-SI-P220

This abstract is available from: http://www.biomedcentral.com/I47I-2202/I0/SI/P220

(c) 2009 Chapochnikov et al; licensee BioMed Central Ltd.

It has been known for more than 30 years that the sensitivity of auditory nerve fibers in mammals is correlated to their spontaneous firing rate and that the variability of these two properties is linked to the auditory system's capability to reliably encode sounds with intensities spanning about 4 orders of magnitude [1]. However, neither the origin nor the coupling of these properties is fully understood. The key players at this early level of the auditory system are the presynaptic active zone of inner hair cells, the postsynaptic spiral ganglion neurons and efferent synapses onto spiral ganglion neurons.

With experiments, we demonstrate pronounced variability of presynaptic $\mathrm{Ca}^{2+}$ signals among different ribbon synapses within single hair cells, e.g. in terms of microdomain amplitude and voltage-dependence. We use modeling to investigate how each of these components could contribute to modify the spontaneous rate and sensitivity. On the presynaptic side, the effect of varying $\mathrm{Ca}^{2+}$ channel number, gating properties and density has been explored. On the postsynaptic side, we vary active and passive membrane properties in the afferent fiber, as well as the strength of a tonically active or intensity-driven feedback inhibition by the efferent fiber.
Parameters for the modeling of the hair cell ribbon synapse are restrained by measurements from in-vivo and invitro experiments, including fast confocal imaging of synaptic calcium signals, current fluctuation analysis derived calcium channel counts, STED-microscopy based calcium channel cluster size estimates, as well as auditory nerve firing properties from single unit recordings. Although not frequently considered in the literature, presynaptic parameters are found capable of strongly influence spontaneous rate and sensitivity.

\section{References}

I. Liberman MC: Auditory-nerve response from cats raised in a low-noise chamber. J Acoust Soc Am 1978, 63:442-455. 